ARQGA / 957

\title{
HIPERTENSÃO PORTAL POR ESQUISTOSSOMOSE MANSÔNICA HEPATOESPLÊNICA: efeito da desconexão ázigo-portal com esplenectomia no diâmetro e na velocidade média de fluxo do sistema portal (estudo ultra-sonográfico com Doppler)
}

\author{
Azzo WIDMAN ${ }^{1}$, Ilka Regina Souza de OLIVEIRA ${ }^{2}$, Manlio Basilio SPERANZINI ${ }^{3}$, \\ Giovanni Guido CERRI ${ }^{4}$, William Abrão SAAD ${ }^{5}$ e Joaquim GAMA-RODRIGUES ${ }^{6}$
}

RESUMO - Racional - A esplenectomia com desconexão ázigo-portal tem sido indicada para o tratamento da hemorragia digestiva pelas varizes esofágicas na hipertensão portal da esquistossomose mansônica hepatoesplênica. Todavia, esta técnica terapêutica apresenta índices variáveis de complicações trombóticas precoces do sistema portal (13,3\% a 53,2\%). Supondo que as alterações circulatórias devidas ao tratamento cirúrgico tenham papel preponderante neste acontecimento, procurou-se identificar elementos hemodinâmicos que, dentre os múltiplos fatores causais, tenham facilitado a ocorrência desta complicação. Com este intuito estudou-se comparativamente, mediante ultra-sonografia com Doppler, o sistema portal de dois grupos de pacientes em condições clínicas semelhantes: não-operados e com desconexão ázigo-portal em fase pósoperatória tardia (periodo superior a 6 meses). Casuística/Método - Foram estudados 58 pacientes com esquistossomose mansônica hepatoesplênica e com antecedentes de hemorragia digestiva alta, divididos em dois grupos: A (29 sob controle ambulatorial: clínico e endoscópico); B (29 submetidos previamente a desconexão ázigo-portal). Em todos foi feita a medida do diâmetro e da velocidade média de fluxo do sangue na veia porta e seus ramos direito e esquerdo, mediante ultrasonografia com Doppler. Os resultados foram submetidos a análise univariada inter e intragrupo. Resultados - No grupo A (não-operados): a veia porta apresentou diâmetro maior do que o dos ramos direito e esquerdo e nestes esta medida foi semelhante (10,6 2,9, 8,0 $\pm 1,8,9,1 \pm 2,6 \mathrm{~cm})$; a velocidade média de fluxo na veia porta e nos ramos portais foi semelhante $(15,62 \pm 6,17,14,92 \pm 5,33,16,12 \pm 4,18 \mathrm{~cm} / \mathrm{seg})$. No grupo B (operados): houve diminuição de ambos os parâmetros na veia porta e seus ramos $(8,8 \pm 1,7,5,2 \pm 1,2,7,5 \pm 2,2 \mathrm{~cm} / 12,53 \pm 2,60,8,86 \pm 1,75,9,60 \pm 3,75 \mathrm{~cm} / \mathrm{seg})$. Conclusões - Houve redução significativa do diâmetro e da velocidade média de fluxo sangüíneo no sistema portal, no pós-operatório tardio, em pacientes com esquistossomose mansônica hepatoesplênica, submetidos a desconexão ázigo-portal.

DESCRITORES - Hipertensão portal. Esquistossomose mansoni. Ultra-sonografia Doppler. Esplenectomia.

\footnotetext{
Trabalho realizado nos Departamentos de Gastroenterologia e Radiologia da Faculdade de Medicina da Universidade de São Paulo - FMUSP.

Médico Assistente Doutor do Grupo de Cirurgia do Fígado e Hipertensão portal da Divisão de Clínica Cirúrgica II do Hospital das Clínicas da FMUSP - HC-FMUSP.

Médica Assistente Doutora do Departamento de Radiologia do HC-FMUSP. Chefe da Ultra-sonografia.

Professor Associado da Disciplina de Cirurgia do Aparelho Digestivo da FMUSP.

Professor Titular do Departamento de Radiologia da FMUSP. Diretor do Instituto de Radiologia do HC-FMUSP.

Livre-Docente do Departamento de Gastroenterologia da FMUSP. Diretor Técnico do Grupo de Cirurgia do Fígado e Hipertensão Portal do HC-

FMUSP. Professor Titular de Cirurgia da Faculdade de Medicina de Sorocaba da Pontifícia Universidade Católica de São Paulo - PUCSP.

Professor Associado e Chefe da Disciplina de Cirurgia do Aparelho Digestivo do Departamento de Gastroenterologia da FMUSP.

Endereço para correspondência: Dr. Azzo Widman - Rua Guilherme Moura, 302 - Vila Ida - 05449-010 - São Paulo, SP.
} 


\section{INTRODUÇÃO}

A hemorragia digestiva é a maior ameaça à sobrevivência dos pacientes com hipertensão portal por esquistossomose mansônica hepatoesplênica, pois a insuficiência hepática é improvável pelas discretas alterações da função hepática.

A desconexão ázigo-portal com esplenectomia (DAPE), complementada posteriormente com escleroterapia endoscópica das veias submucosas do esôfago, tem proporcionado bons resultados clínicos no tratamento desta complicação hemorrágica ${ }^{(21)}$. Entretanto, esta terapia cirúrgica tem sido acompanhada da ocorrência freqüente de trombose pós-operatória do sistema portal $\left(13,3 \%^{(4)}\right.$ a $\left.53,2 \%{ }^{(19)}\right)$, algumas vezes de natureza grave $\mathrm{e}^{(5)} \mathrm{e}$ cuja etiopatogenia ainda não está bem esclarecida.

A esplenectomia, eliminando o fluxo sangüíneo esplênico, e a desconexão, interrompendo a via de escape cardiotuberositária, causam situação hemodinâmica complexa no sistema portal, que pode concorrer para o fenômeno trombótico na fase pós-operatória.

Dos fatores circulatórios intervenientes, a redução da pressão portal, demonstrada pela diminuição do calibre da veia porta ${ }^{(19)}$, possivelmente tem papel preponderante na multifatoriedade causal desta complicação, mas a velocidade circulatória do sangue portal também atua neste contexto e deve ser considerada. Este parâmetro hemodinâmico, que não tem sido suficientemente valorizado na esquistossomose hepatoesplênica, é interdependente da pressão neste território, pois se esta representa o estímulo para a hipertrofia das vias colaterais, a velocidade de fluxo é influenciada diretamente pela circulação através dos vasos assim formados e/ou dilatados ${ }^{(*)}$.

Com base nestas considerações, resolveu-se avaliar, mediante ultrasonografia com Doppler (US-Doppler), a circulação portal (diâmetro e velocidade média de fluxo da veia porta) de dois grupos de doentes, em estágio semelhante da moléstia, não-operados e com DAPE (pósoperatório tardio - período superior a 6 meses).

Esta decisão se baseia na suposição de que as alterações hemodinâmicas detectáveis no pós-operatório tardio, sejam o retrato fiel, embora amenizado, das mudanças induzidas pelo ato cirúrgico e que a comparação dos resultados dos exames destes pacientes possa oferecer subsídios que contribuam para esclarecer a situação circulatória que a DAPE provoca no sistema portal.

\section{CASUÍSTICA E MÉTODO}

Foram estudados 58 pacientes com esquistossomose hepatoesplênica, seqüenciais, do Ambulatório do Serviço de Cirurgia do Fígado e Hipertensão Portal do Hospital das Clínicas da Universidade de São Paulo, que foram divididos em dois grupos:

Grupo A - 29 doentes com antecedentes de sangramento digestivo prévio, não-operados, em acompanhamento clínico e endoscópico.
Grupo B - 29 doentes submetidos a DAPE devido a sangramento prévio através de varizes esofágicas.

Exame ultra-sonográfico - Todos os pacientes foram examinados por US-Doppler, em condições de jejum de 12 horas e ingestão prévia de $250 \mathrm{mg}$ de dimeticona (Luftal@) para reduzir a interferência dos gases intestinais.

Os exames foram realizados em equipamento de ultra-sonografia de tempo real, com transdutor convexo de 3,5-MHz (GE - Logic 500 MD, USA) com Doppler pulsado na freqüência de 3,5 e 2,5 MHz.

Após identificar a veia porta, o ramo portal direito e esquerdo, no modo B, foi realizada medida do diâmetro destes vasos. Em seguida, o feixe Doppler foi posicionado no vaso estudado e utilizada amostra de volume com abertura ("sample volume length") correspondendo a cerca de $2 / 3$ do calibre para medida da velocidade média de fluxo. Esta foi calculada automaticamente mediante "software" específico do equipamento (operador-independente) a partir do traçado espectral Doppler.

Critério de inclusão - diagnóstico prévio de esquistossomose hepatoesplênica, sistema portal pérvio, medidas realizadas em todos os segmentos vasculares estudados.

Critério de exclusão - presença de hepatopatia não-esquistossomótica, trombose de vasos do sistema portal, presença de veia paraumbilical pérvia e com fluxo hepatofugal.

Os valores foram expressos em média e desvio padrão.

Análise estatística - As médias e o desvio padrão dos resultados foram submetidos a análise estatística pelo teste de variância univariada ("one way analysis of variance") com a finalidade de realizar a comparação dos valores inter- e intra-grupo A e B.

\section{RESULTADOS}

A análise comparativa dos resultados intra-grupo mostrou que:

\section{Diâmetro -}

A - pacientes não-operados

1. a veia porta tem diâmetro maior que o ramo portal direito $(P=0,000)$ e ramo esquerdo $(P=0,043)$

2. o diâmetro do ramo direito é equivalente ao do ramo esquerdo $(P=0,066)$

$\mathrm{B}$ - pacientes operados

1. a veia porta tem diâmetro maior que o ramo portal direito $(P=0,000)$ e ramo portal esquerdo $(P=0,015)$

2. o ramo portal esquerdo tem diâmetro maior que o ramo portal direito $(P=0,000)$

\section{Velocidade média de fluxo -}

A - pacientes não-operados: há eqüivalência nas velocidades média de fluxo na veia porta $(P=0,646)$, ramo portal direito $(P=0,719)$ e no ramo portal esquerdo $(P=0,344)$

* Oliveira IRS. Comunicação pessoal. 2000. 
B - pacientes operados: a velocidade média de fluxo na veia porta é maior do que no ramo portal direito $(P=0,000)$ e ramo portal esquerdo $(P=0,000)$ onde ela é eqüivalente $(P=0,340)$

A análise comparativa dos resultados inter-grupos mostrou que:

Diâmetro - houve redução do diâmetro da veia porta $(P=0,0006)$, do ramo direito $(P=0,000)$ e do ramo esquerdo $(P=0,014)$ no pósoperatório.

Velocidade média de fluxo - houve redução da velocidade média de fluxo na veia porta $(P=0,016)$, no ramo direito da veia porta $(P=0,000)$ e ramo esquerdo da veia porta $(P=0,000)$ no pósoperatório.

Estes resultados estão resumidos nas Tabelas 1 e 2.

\section{COMENTÁRIOS}

Na esquistossomose hepatoesplênica, a hipertensão portal é provocada pelo bloqueio pré-sinusoidal intra-hepático e sua intensidade é resultante de dois fatores circulatórios que se opõem: aporte venoso esplênico e mesentérico (com papel preponderante) e eficiência do escape do sangue portal pelas várias vias de circulação colateral.

Desse modo, do ponto de vista exclusivamente hemodinâmico, a operação "ideal" deveria reduzir o aporte sangüíneo - por exemplo, eliminando o importante contingente venoso esplênico - e facilitar o escoamento do sangue portal pelas vias colaterais (naturais ou artificialmente construídas).

A DAPE preenche a primeira parte dessa premissa (eliminação do contingente venoso esplênico), mas o escoamento do sangue portal pelas vias colaterais naturais é dificultado pela desconexão ázigoportal (interrupção do aporte venoso às varizes esofágicas).
Assim, da inter-relação dos efeitos circulatórios destas duas ações cirúrgicas, deriva uma situação hemodinâmica complexa que procuramos avaliar. Com esta finalidade, analisaram-se os parâmetros mensurados, pela US-Doppler, dos vasos do sistema portal: diâmetro e velocidade média de circulação.

Este método de imagem possibilitava, inicialmente, a medida do diâmetro de vasos portais mas, ao ser associada ao Doppler colorido e pulsado, permitiu que se avaliassem, também, a direção e a velocidade do fluxo sangüíneo neste sistema ${ }^{(14)}$.

Desde sua introdução na prática médica, este exame tem sido utilizado para o estudo de várias doenças hepáticas e para criar índices

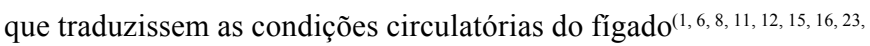
24, 26).

Progressivamente, dificuldades relacionadas ao método foram sendo reconhecidas e equacionadas para que esta técnica pudesse ser utilizada eficientemente no estudo deste sistema vascular ${ }^{(3,7,20,22,27,28)}$.

Sua importância no estudo da síndrome hipertensiva portal ficou estabelecida quando, apesar das dificuldades inerentes ao método, foi

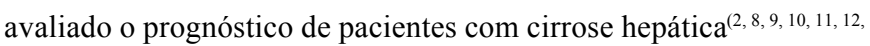
13, 17, 18, 25, 26, 29, 30).

Tais estudos, entretanto, foram realizados predominantemente em pacientes portadores de cirrose hepática, mas suas contribuições podem ser estendidas à investigação fisiopatológica do sistema portal na fibrose hepática esquistossomótica.

\section{Análise do diâmetro dos vasos portais}

No presente estudo, a análise intragrupos (Tabelas 1,2) mostra o fato anatomicamente previsível de que em ambos os grupos (operados e não-operados) o calibre da veia porta é maior do que o

TABELA 1 - Aspectos comparativos dos diâmetros dos vasos portais de doentes não-operados e operados de DAPE

\begin{tabular}{lcc}
\hline & \multicolumn{3}{c}{ Diâmetro $(\mathrm{mm})$} \\
\hline Veia porta (tronco) & Grupo A (não-operados) $\mathbf{n}=\mathbf{2 9}$ & Grupo B (DAPE) $\mathbf{n}=\mathbf{2 9}$ \\
Ramo portal direito & $10.6 \pm 2.9$ & $8.8 \pm 1.7$ \\
Ramo portal esquerdo & $8.0 \pm 1.8$ & $5.2 \pm 1.2$ \\
\hline
\end{tabular}

DAPE: desconexão ázigo portal

TABELA 2 - Aspectos comparativos da velocidade média de fluxo nos vasos portais de pacientes não-operados e operados de DAPE

\begin{tabular}{lcc}
\hline & \multicolumn{2}{c}{ Velocidade média de fluxo (cm/seg) } \\
\hline Grupo A (não-operados) $\mathbf{n = 2 9}$ & Grupo B (DAPE) n=29 \\
\hline Veia porta (tronco) & $15.62 \pm 6.17$ & $12.53 \pm 2.60$ \\
Ramo portal direito & $14.92 \pm 5.33$ & $8.86 \pm 1.75$ \\
Ramo portal esquerdo & $16.12 \pm 4.18$ & $9.60 \pm 3.75$ \\
\hline
\end{tabular}

DAPE: desconexão ázigo portal 
dos seus ramos. Entretanto, a mesma análise mostra que, nos casos operados, há redução estatisticamente significativa, comparativamente aos não-operados, embora seja mantida a relação já observada (Tabela 1). Esta diminuição do calibre no pós-operatório relaciona-se com a baixa da pressão portal provocada pela esplenectomia ${ }^{(19)}$, mas como não há alteração do bloqueio intrahepático, era esperado que, na fase tardia, com o reajustamento da situação circulatória, houvesse retorno às condições iniciais. A redução a longo prazo do calibre dos vasos portais (Tabela 1), se considerarmos este parâmetro confiável, indicaria que não houve restabelecimento da pressão portal em nível anterior.

\section{Análise da velocidade média de fluxo no sistema portal}

A redução da velocidade de fluxo sangüíneo, observada na veia porta dos pacientes operados (Tabela 2), tem implicações mais complexas, pois depende da tríade: bloqueio intra-hepático, aporte e drenagem do sangue portal.

A DAPE não modifica o bloqueio intra-hepático, mas os atos cirúrgicos que a compõem interferem na circulação portal: a esplenectomia influencia a pressão portal, eliminando o território esplênico e a desconexão ázigo-portal atua diretamente na drenagem do sangue portal. Neste procedimento, a ligadura cirúrgica das comunicações venosas extramurais do esôfago junto com a vertente vascular gástrica esquerda reduz o escape, pois interrompe esta via circulatória portossistêmica retroperitonial e provoca aumento do volume a montante. Mesmo sendo desconhecido o contingente circulatório drenado por esta via, o sangue que passa a ser represado no sistema portal pode contribuir para a lentidão circulatória no território mesentérico-portal. Esta queda acentuada e sustentada da velocidade média de fluxo na veia porta e seus ramos, como se pôde comprovar, não é contrabalançada completamente pela diminuição da oferta circulatória que resulta da esplenectomia.

Deste modo, junto com a diminuição da pressão portal, inferida pela redução do diâmetro dos vasos portais, este parâmetro pode ser considerado fator hemodinâmico importante no agravamento e na perpetuação das complicações vasculares pós-operatórias no sistema portal destes pacientes.

Concluindo, verificaram-se que as observações acima (redução do calibre das veias do sistema portal e queda da velocidade média de fluxo), levam a admitir que, embora a etiopatogenia das tromboses pós-operatórias do sistema portal seja multifatorial, a mudança de regime circulatório, como foi demonstrado, pode ter papel preponderante, criando condições propícias para a coagulação intravascular, o que justificaria a alta incidência desta complicação na fase pós-operatória imediata.

Widman A, Oliveira IRS de, Speranzini MB, Cerri GG, Saad WA, Gama-Rodrigues J. Hepatosplenic schistosomotic portal hypertension: effect of esophagogastric devascularization with splenectomy on the diameter and mean flow velocity in the portal system (ultra-sonographic Doppler study). Arq Gastroenterol 2001;38(1):19-23.

ABSTRACT - Background - Esophagogastric devascularization with splenectomy has been used for the treatment of upper digestive bleeding due to esophagic varices in hepatoportal mansoni's schistosomic portal hypertension. Nevertheless, early portal thrombosis has hampered this surgical technique (13,3\% and 53,2\%), compromising the good results on the hemorrhagic side. Supposing that portal circulatory changes, due to the surgical treatment, may play an important role in this kind of complication, our objective was to identify the hemodynamic facilitating factors. Portal hemodynamic aspects, identified by ultra-sonographic Doppler study, from two groups of patients: nonoperated upon and splenectomized with esophagogastric devascularization in late post-operatory phase (in excess of 6 moths), with portal hypertension due to mansoni hepatoesplenic portal hypertension and in similar clinical conditions, were compared. Method - Fifty eight ambulatorial patients were studied, all had portal hypertension caused by mansoni's hepatosplenic schistosomiasis and previous bouts of digestive bleeding. They were divided in two groups: A - 29 followed clinically/endoscopically, and group B - 29 previously submitted to esophagogastric devascularization with splenectomy. In all was measured the diameter and mean flow velocity in the portal vein and its right and left branches by ultra-sonographic Doppler study. The results were submitted to statistical analysis for inter-and intra-group comparison. Results - Group A (non-operated): the portal vein diameter was greater than the right and left branches (10.6 $\pm 2.9,8.0 \pm 1.8$, $9.1 \pm 2.6 \mathrm{~cm})$, the mean flow velocities in the portal vein and its branches were similar $(15.62 \pm 6.17,14.92 \pm 5.33,16.12 \pm 4.18 \mathrm{~cm} / \mathrm{seg})$. Group B (operated): the diameter and mean flow velocity in all vessels were reduced $(8.8 \pm 1.7,5.2 \pm 1.2,7.5 \pm 2.2 \mathrm{~cm} / 12.53 \pm 2.60,8.86$ $\pm 1.75,9.69 \pm 3.75 \mathrm{~cm} / \mathrm{seg}$ ). Conclusions - After esophagogastric devascularization with splenectomy, there was a reduction of the diameter and mean flow velocity in the portal vein, its right and left branches.

HEADINGS - Hypertension, portal. Schistosomiasis mansoni. Ultrasonography, Doppler. Splenectomy. 


\section{REFERÊNCIAS BIBLIOGRÁFICAS}

1. Aubé C, Oberti F, Korali N, Namour M-A, Loisel D, Tanguy J-Y, Valsesia E, Pilette C, Rousselet MC, Bedossa P, Rifflet H, Maïga MY, Penneau-Fontbonne $\mathrm{D}$, Caron C, Calès P. Ultrasonographic diagnosis of hepatic fibrosis or cirrhosis. J Hepatol 1999;30:472-8.

2. Blanco MBD, Dios Vega JF, Miño Fugarolas G. Contribuición de la ecografía Doppler a la historia natural dela hipertensión portal. Rev Esp Enferm Dig 1998;90:767-70.

3. Bolondi L, Gaiani S, Barbara L. Accuracy and reproducibility of portal flow measurements by Doppler US. J Hepatol 1991;13:269-73.

4. Chaib E, Pugliesi V, Capacci ML, D'Albuquerque LC, Widman A, Bernardini AP, Oliveira e Silva A, Saad WA, Machado MCC, Pinotti HW. Trombose portal: complicação precoce da desconexão ázigo-portal no tratamento das varizes esofagianas sangrantes. Rev Hosp Clin Fac Med S Paulo 1990;45:205-7.

5. Chaib E, Herman P, D'Albuquerque LC, Pugliesi V, Antonio LGM, Feijó LFA, Ishida RY, Saad WA, Pinotti HW. Trombose mesentérica após desconexão ázigoportal com esplenectomia para tratamento das varizes esofagianas sangrantes na esquistossomose mansônica, relato de três casos. Rev Hosp Clin Fac Med S Paulo 1996;51:65-8.

6. Gaiani S, Bolondi L, Bassi SL, Zironi G, Siringo S, Barbara L. Prevalence of spontaneous hepatofugal portal flow in liver cirrhosis. Gastroenterology 1991;100:160-7.

7. Gibson RN, Gibson PR, Donlan JD, Padmanabhan R. Modified Doppler flowmetry in the splancnic circulation. Gastroenterology 1993;105:1029-34.

8. Haag K, Rössle M, Ochs A, Huber M, Siegerstetter V, Olschewsky M, Berger E, Lu S Blum HE. Correlation of Duplex sonography findings and portal pressure in 375 patients with portal hypertension. AJR Am J Roentgenol 1999;172:631-5.

9. Iwao T, Toyonaga A, Oho K, Sakai T, Tayama C, Masumoto H, Sato M, Nakahara $\mathrm{K}$, Tanikawa K. Postprandial splancnic hemodynamic response in patients with cirrhosis of the liver: evaluation with "triple-vessel" duplex US. Radiology 1996;201:711-5.

10. Iwao T, Toyonaga A, Shigemori H, Oho K, Sakai T, Tayama C, Masumoto H, Sato $\mathrm{M}$, Tanikawa K. Hepatic artery hemodynamic responsiveness to altered portal blood flow in normal and cirrhotic livers. Radiology 1996;200:793-8.

11. Iwao T, Toyonaga A, Oho K, Tayama C, Masumoto H, Sakai T, Sato M, Tanikawa $\mathrm{K}$. Value of Doppler ultrasound parameters of portal vein and hepatic artery in the diagnosis of cirrhosis and portal hypertension. Am J Gastroenterol 1997;92:1012-7.

12. Kok Th, Jagt EJ, Haagsma EB, Bijveld CMA, Jansen PLM, Boeve WJ. The value of Doppler ultrasound in cirrhosis and portal hypertension. Scand J Gastroenterol 1999;(Suppl 230):82-8.

13. Körner T. Portal duplex sonography in liver cirrhosis - A useful supplement to endoscopic evaluation of bleeding risk of esophageal varices? Scand J Gastroenterol 1996;31:495-9.

14. Moriyasu F, Ban N, Nishida O, Nakamura T, Miyake T, Uchino H, Kanematsu Y, Koizumi S. Clinical application of an ultrasonic duplex system in the quantitative measurement of portal blood flow. J Clin Ultrasound 1986;14:579-88

15. Moriyasu F, Nishida O, Ban N, Nakamura T, Miura K, Sakai M, Miyake T, Uchino H. Measurement of portal vascular resistance in patients with portal hypertension. Gastroenterology 1986;90:710-7.
16. Moriyasu F, Nishida O, Ban N, Nakamura T, Sakai M, Miyake T, Uchino H. "Congestion Index" of the portal vein. AJR Am J Roentgenol 1986;146:735-9.

17. Paulson EK, Kliewer MA, Frederick MG, Keogan MT, DeLong DM, Nelson RC. Doppler US mesurement of portal venous flow: variability in healthy fasting volunteers. Radiology 1997;202:721-4.

18. Pérez F, Garcia Montes JM, Castro Laria L, Martín Guerrero JM, Jiménez Sáenz M, Herrerías Gutiérrez JM. Valor de la ecografía en el diagnóstico de la hipertension portal. Rev Esp Enferm Dig 1998;90:806-12.

19. Pugliesi V. Desconexão ázigo-portal e esplenectomia associadas à esclerose endoscópica no tratamento das varizes do esôfago na esquistossomose hepatoesplênica [Tese de Doutorado]. São Paulo: Faculdade de Medicina da Universidade de São Paulo; 1996. p.38.

20. Sabbá C, Weltin GG, Cicchetti DV, Ferraioli G, Taylor KJW, Nakamura T, Moriyasu F, Groszmann RJ. Observer variability in echo-Doppler measurements of portal flow in cirrhotic patients and normal volunteers. Gastroenterology 1990;98:1603-11.

21. Sakai P, Boaventura S, Ishioka S, Mies S, Sette H, Pinotti HW. Sclerotherapy of bleeding esophageal varices in schistosomiasis - comparative study in patients with and without previous surgery for portal hypertension. Endoscopy 1990;22:5-7.

22. Scheurlen M, Waller C, Hartman F. In vivo validation of image-directed Doppler measurement of superior mesenteric artery blood flow. J Clin Ultrasound 1992;20:19-26

23. Schneider AW, Kalk JF, Klein CP. Hepatic arterial pulsatility index in cirrhosis: correlation with portal pressure. J Hepatol 1999;30:876-81.

24. Sinha R. Grayscale and pulsed Doppler. Characteristics of non-cirrhotic portal fibrosis: a preliminary report. Clin Radiol 1999;54:156-9.

25. Siringo S, Bolondi L, Gaiani S, Sofia S, Zironi G, Rigamonti A, Febo G, Migliol M, Cavalli G, Barbara L. Timing of the first variceal hemorrhage in cirrhotic patients: prospective evaluation of Doppler flowmetry, endoscopy and clinical parameters. Hepatology 1994;20:66-73.

26. Taourel P, Blanc P, Dauzat M, Chabre M, Pradel J, Gallix B, Larrey D, Bruel J-M. Doppler study of mesenteric, hepatic and portal circulation in alcoholic cirrhosis: relationship between quantitative Doppler measurements and the severity of portal hypertension and hepatic failure. Hepatology 1998;28:932-6.

27. Vries PJ, Hattum J, Hoekstra JBL, Hooge P. Duplex Doppler measurements of portal venous flow in normal subjects - inter- and intra-observer variability. $\mathrm{J}$ Hepatol 1991;13:358-63.

28. Zironi G, Gaiani S, Fenyves D, Rigamonti A, Bolondi L, Barbara L. Value of measurement of mean portal flow velocity by Doppler flowmetry in the diagnosis of portal hypertension. J Hepatol 1992;16:298-303.

29. Zoli M, Iervese T, Merkel C, Bianchi G, Magalotti D, Marchesini G, Gatta A, Pis E. Prognostic significance of portal hemodynamics in patients with compensated cirrhosis. J Hepatol 1993;17:56-61.

30. Zoli M, Magalotti D, Bianchi G, Ghigi G, Orlandini C, Grimaldi M, Marchesin G, Pisi E. Functional hepatic flow and Doppler-assessed total hepatic flow in control subjects and in patients with cirrhosis. J Hepatol 1995;23:129-34.

Recebido em 9/5/2000 Aprovado em 21/9/2000 